\title{
Ethno-Gynaeacological Knowledge and Preliminary Phytochemical Screenings of Medicinal Plants used in Lagos State, Nigeria
}

\author{
OJ Sharaibi*, DA Adeogun, OT Abati \\ Department of Botany, Lagos State University, Ojo, Lagos State, Nigeria
}

*Corresponding Author: OJ Sharaibi, Department of Botany, Lagos State University, Ojo, Lagos State, Nigeria

\begin{abstract}
Gynaeacological disorders are various diseased conditions negatively affecting female reproductive organs. This study was conducted to establish a regional profile of the indigenous knowledge on the treatment of various gynaeacological disorders in Lagos State, Nigeria. Oral and semi-structured interviews were used to obtain information from 100 local informants in five local government areas of Lagos State, Nigeria. Qualitative phytochemical screenings of the medicinal plants were done using standard laboratory procedures. Fifty (50) plant species belonging to 35 families were identified for the treatment of different gynaeacological disorders in the study area. Ethnobotanical uses of 16 plant species for amenorrhea, 9 species for aphrodisiac, 7 species for vaginal infections and 6 plant species for sexually transmitted diseases were discovered in the study area. Leaves were the most commonly used plant part (29.03\%) followed by the bark (22.58\%) while the least commonly used plant part was the bulb (1.61\%). Decoction (48.08\%) was commonly used mode of preparations, followed by powdered form (23.08\%) while taking the plant raw (1.29\%) was the least commonly used method. Phenols and flavonoids were present in all the plants while steroids were present in all the plants except six plants and phlobatannins were present only in ten (10) plant species. This study showed that Lagos State is rich in plant species that contain significant bioactive compounds useful in the treatment of various gynaeacological disorders in Lagos State, Nigeria.
\end{abstract}

Keywords: Gynaeacological disorders, Lagos State, Ethnobotanical survey, Medicinal plants, Phytochemicals

\section{INTRODUCTION}

The prevalence of gynaeacological disorders worldwide has given rise to increased female infertility, morbidity and mortality (Ikechebelu, 2005). Gynaeacological disorders are various diseases and conditions negatively affecting female reproductive organs. They can be minor and easily treated or devastating. They negatively impact woman's quality of life, fertility and longevity. Uterine fibroids, annovulation, amenorrhea, dysmenorrhea, endometriosis, hyperprolactinemia, pelvic inflammatory disease, dyspareunia, lactation problems, delivery problems, miscarriages, sexually transmitted diseases, tubal damage and gynaeacological cancers are some of the common gynaeacological disorders affecting women in Nigeria and the world at large. These can be as a result of several factors which may be physical, pathological and pharmacological. Pandey and Bhattacharya (2010) stated that physical factors such as age, stress, poor diet, lack of exercise, overweight, underweight and obesity can lead to abnormal functioning of the female reproductive system. Pathological causes are diseases of hypothalamus, pituitary, thyroid, adrenal glands, ovarian disorders, congenital disorders of genital organs and chromosomal abnormalities (Freeman et al., 2000). Certain medications such as reserpine, antipsychotic drugs, risperidone, phenothiazines, metoclopramide, oral contraceptive pills etc. have been indicted to be responsible for some gynaeacological disorders (Melmed et al., 2011).

Gynaeacological disorders have been implicated in the aetiology of female infertility currently affecting $30 \%$ or more women of reproductive age (Rustein and Shah, 2004). Eggleston and Victor (2012) reported that, global total fertility in 1960s was 6.1 million, but it has now fallen to 4.2 million. According to Sule et al. (2008), $30 \%$ of women in Nigeria have proven difficulties in achieving pregnancy after two years of contraceptive free intercourse. Hyperprolactinemia (Azima and Samina, 
2002), tubal damage (Akande et al., 2003), reproductive tract infections (Ali et al., 2007), annovulation (Nduche et al., 2015), sexually transmitted diseases (Inyang-Etoh et al., 2009), leucorrhea (LIewellyn-Jones, 1998), menstrual disorders (Dag and Dilbaz, 2015) are some of the factors reported to account for a significant proportion of cases of female infertility.

Gynaeacological disorders have also been associated with gynaeacological morbidity such as foulsmelling vaginal discharge, uterovaginal prolapse post-coital bleeding, heavy menstrual bleeding and tiredness. Symptoms of gynaeacological morbidity were found to have negative impact on health related quality of woman's life (Black and Fraser, 2012). Heavy menstrual bleeding has been linked to anaemia; maternal anaemia is associated with low fetal growth and increase in maternal mortality (Kalaivani, 2009). Gynaeacological cancers are associated with high rate of fatality in women. Endometrial cancer has been reported to be the most common cancer of the female reproductive organs (Jema et al., 2011) while ovarian cancer has been reported to be the commonest cause of death among all gynecological cancers (Hennessy et al., 2009).

The use of conventional medicines in the treatment and management of different gynaeacological disorders has been awesome but the drugs have been associated with gastrointestinal, cardiovascular and neurological side effects (van Rijswijk and Vermorken, 2000). Calao et al. (2006) reported that the use of dopamine agonist drugs such as bromocriptine, carbegoline and pergolide to treat hyperprolactinemia were linked to the development of certain types of hyperplasia in women.

Medicinal plants have been reported to be effective in the treatment of different gynaeacological disorders; Angelica sinensis (Schellenberg, 2001), Vitex agnus-castus (Wuttke et al., 2003), Cimicifuga racemosa (Schellenberg, 2001), Eletherococcus senticosis (Mayo, 1998), Coriandrum sativum (Sadeghi and Mahmood, 2014), Foeniculum vulgare (Sadrefozalayi and Farokhi, 2014) and Cuminum cyminum (Tavasoli et al., 2002) were some of the plants reported. This study therefore, aimed at identifying, documenting and preliminary phytochemical screening of the indigenous medicinal plants used for the treatment of various gynaeacological disorders in Lagos State, Nigeria. This is to establish a regional profile of the indigenous knowledge on the treatment of various gynaeacological problems in Lagos State, Nigeria.

\section{STUDY AREA}

The study was carried out in Agege, Badagry, Ojo, Mushin and Somolu Local Government Areas of Lagos State, Southwestern Nigeria (Figure 1). Lagos State is located on the southwestern geopolitical zone of Nigeria and is the most economically important and the financial centre of the country. It is located on longitude $6^{\circ} 35^{\prime} \mathrm{N}$ and latitude $3^{\circ} 45^{\prime} \mathrm{E}$ with an elevation of $41 \mathrm{~m}(135 \mathrm{ft})$ above sea level. It occupies a total area is $3,577 \mathrm{~km}^{2}$ with an approximate population of 16 million (Census 2006), $22 \%$ of the total area are lagoons and creeks.

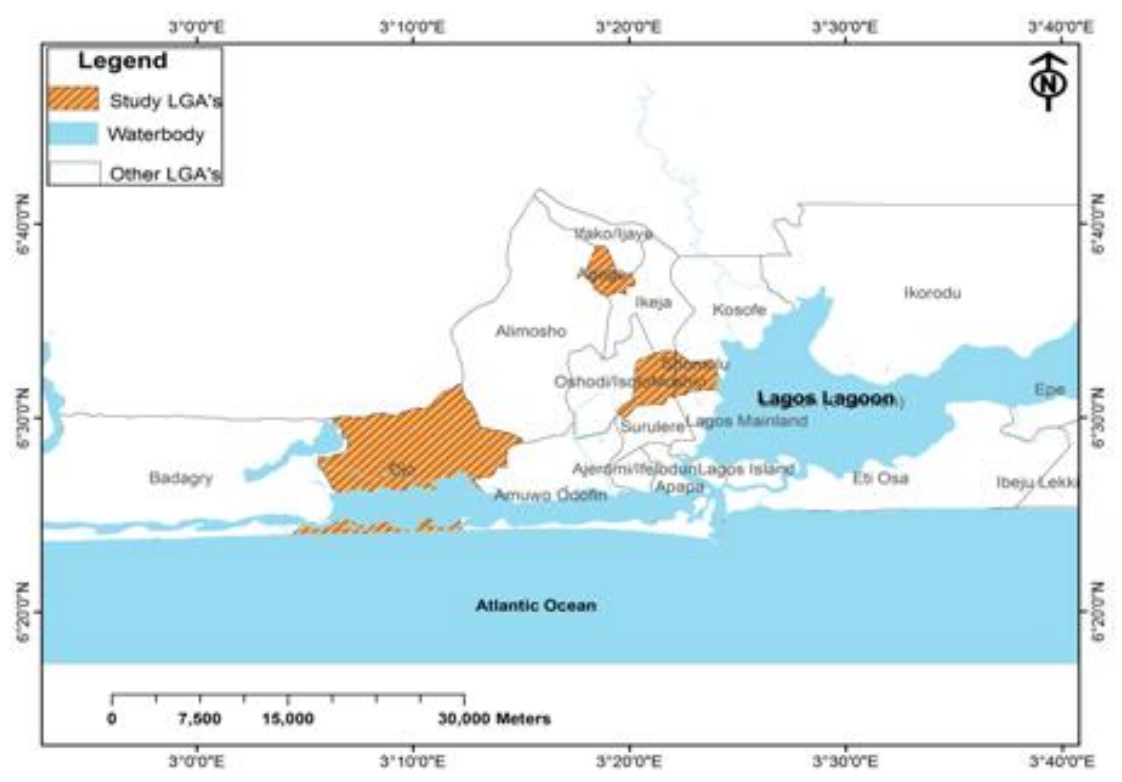

Figure1. Map of Lagos State, Nigeria showing the study area 


\section{Materials AND Methods}

\subsection{Data Collection}

Collection of data was done between September 2014 and June 2015. Ethno medicinal information was obtained through oral and semi-structured interviews. The interviews were conducted randomly with a total of 100 local respondents including herb sellers, herbalists, elderly people and others with the knowledge of herbal medicine (Table 1). The consents of all the respondents were sought before the interviews. The interviews were held in our local language (Yoruba) hence, there was no need for interpreter and this allowed accurate data recordings. The information collected included different gynaeacological disorders commonly affecting women in the study area, plants or plant parts used for the treatment, the local names and modes of preparation and administration of the medicinal plants.

Table1. Distribution of local informants in accordance with their age groups

\begin{tabular}{|l|l|l|l|l|l|}
\hline \multirow{2}{*}{ Age Groups } & \multicolumn{5}{|c|}{ Categories of Informants } \\
\cline { 2 - 6 } & Herb sellers & Herbalists & Elderly people & Others & Total \\
\hline $31-40$ & 5 & - & - & 1 & 6 \\
\hline $41-50$ & 21 & 7 & - & 3 & 31 \\
\hline $51-60$ & 10 & 2 & 3 & 4 & 19 \\
\hline $61-70$ & 10 & 2 & 4 & 6 & 22 \\
\hline 71 and above & 5 & 5 & 10 & 2 & 22 \\
\hline Total & 51 & 16 & 17 & 16 & 100 \\
\hline
\end{tabular}

\subsection{Collection and Identification of Medicinal Plants}

Fresh plant materials were collected from the study area. They were identified by Dr. OJ Sharaibi, a plant taxonomist and the voucher specimens were deposited in Lagos University Herbarium for reference.

\subsection{Sample Preparation and Extraction}

The fresh plant materials were rinsed, air dried and pulverized before extraction. One hundred gram each of the powdered material was soaked in $1000 \mathrm{~mL}$ of distilled water. The extracts were filtered through Whatman no.1 paper and the filtrate was freeze-dried for $48 \mathrm{~h}$ using a freeze dryer (Vir Tis benchtop K, Vir Tis Co., Gardiner, NY).

\subsection{Qualitative Phytochemical Screenings}

The aqueous extract of all the medicinal plants used in folklores for the treatment of various gynaeacological disorders in Lagos State, Nigeria were screened for the presence of secondary metabolites using standard laboratory procedures (Trease and Evans, 2002; Harborne, 2005).

\subsection{Data Analysis}

Simple calculations and quantification of the ethno-botanical data was done by using the relative frequency citation (RFC) technique. This was obtained by dividing the frequency of citation of a plant species (FC) by the total number of species reported.

$R F C=F C / N$

The percentage relative frequency ratio was obtained using the formula:

$$
\% R F C=F C / N X 100
$$

Where FC is the number of times a particular species is mentioned, and $\mathrm{N}$ is the number of all respondents.

\section{RESUltS AND DISCUSSION}

\subsection{Details of the Informants}

Table 1 showed the profile of the local respondents interviewed for the medicinal plants used for the treatment of gynaeacological disorders in Lagos State, Nigeria. 
One hundred local informants were interviewed during this study; 51 herb sellers, 17 elderly people, 16 herbalists and 16 others with knowledge of herbal medicine. This showed the availability of indigenous knowledge of herbal medicine in Lagos State that is majorly a metropolitan state. Majority of the informants were between 40-50 years of age (31\%). This study revealed that parents are the major custodians of the herbal knowledge being transferred to the younger generation and little or none is learnt from Schools. The respondents especially the herb sellers and the herbalists claimed that most women in the study area with gynaeacological disorders preferred traditional medicine to orthodox medicine due to the high rate of consultation. WHO (2002) stated that $80 \%$ of people in developing countries rely on herbal medicine for their primary health care. The preference for herbal medicine in the study area can be attributed to poverty, accessibility to medicinal herbs and general belief that medicinal herbs are less toxic. The positive attitude of the informants to divulge information on the local herbs used for various gynaeacological disorders in the study area was an added advantage in achieving the aim of this study.

\subsection{Medicinal Plants used for Gynaeacological Disorders}

Fifty plant species belonging to 35 families were identified for the treatment of various gynaeacological disorders in five (5) Local Government Areas of Lagos State, Nigeria (Table 2). The distribution of the medicinal plants used for gynaeacological disorders within the families as shown in Table 3 revealed that Fabaceae had the highest number of plant species $(10 \%)$ followed by Euphorbiaceae $(8 \%)$. Annonaceae and Poaceae had three species each $(6 \%)$ this was followed by Anarcadiaceae, Bignoniaceae and Vitaceae with two species each (4\%). The remaining families had one species each. Most of these plants were edible while some were used mainly for medicinal purposes. Sixteen (16) out of the fifty (50) medicinal plants identified were used for the treatment of amenorrhea in the study area. This may be an indication that, the most common gynaeacological disorder affecting women in the study area was amenorrhea. Amenorrhea can be the resultant effects of other gynaeacological disorders such as hyperprolactinemia, hormonal imbalance, ovarian cysts, endometriosis and pelvic inflammatory disease ( Fourman and Fazeli, 2015). Nine (9) plant species were identified as aphrodisiac and libido enhancer while seven (7) plant species were identified for the treatment of sexually transmitted diseases. One plant each was identified for the treatment of tubal blockage, endometriosis and ovarian cysts. This may suggest that these disorders were not common in the study area or the informants do not know the herbal remedies for these disorders (Figure 2). Some of the plant species identified were used to treat more than one gynaeacological disorders; Aloe vera was used as an aphrodisiac and also to treat amenorrhea as well as vaginal infections. Lawsomia inermis was used for the treatment of gonorrhea, leucorrhea and menorrhagia. This may be due to the presence of several bioactive compounds in these plants that can exhibit significant therapeutic actions on various gynaeacological disorders. Some of the plants mentioned have been reported to be used in herbal medicine for the treatment of other disease conditions. Mangifera indica (Titanji et al., 2008), Morinda lucida (Odugbemi et al., 2007) and Azadirachta indica (Alzohairy, 2016) are used for the treatment of malaria. Heliotropium indicum is used to treat skin infections and wounds (Muthu et al., 2006), Ocimum sanctum for arthritis, dysentery and diarrhea (Pattanayak et al., 2010) and Zingiber officinale for cold, flu and hypertension ( Al-Nahain et al., 2014).

Earlier survey reported that Acacia nilotica, Calotropis procera, Citrullus colocynthis, Kigellia Africana, Morinda lucida, Mucuna pruriens, Nymphaea lotus, Plumbago zeylanica, Spondias mombin, Xylopia aethiopca and Zingiber officinale are used for the treatment of infertility in Ogun State, Nigeria (Soladoye et al., 2014). Similarly, Nduche et al. (2015) mentioned Mucuna pruriens, Uvaria chamae, Xylopia aethiopica, Newbouldia laevis, Kigellia Africana, Zingiber officinale, Chochorus olitorius, Citrullus colocynthis, Heliotropium inducm, Anthocleista djalonensis, Viscum album and Azadirachta indica in an ethnobotanical survey for the treatment of fertility problems in Ebonyi state, Nigeria. Nymphaea lotus, Uvaria chamae and Anthocleista djalonensis have also been reported by Sharaibi et al. (2014) for the treatment of hyperprolactinemia in Southwestern, Nigeria. This confirms the ethno-gynaeacological uses of the plants mentioned by the respondents in the study area. 
Ethno-Gynaeacological Knowledge and Preliminary Phytochemical Screenings of Medicinal Plants used in Lagos State, Nigeria

Table2. Medicinal Plants used for the Treatment of Gynaeacological Disorders in Lagos State, Nigeria

\begin{tabular}{|c|c|c|c|c|c|c|}
\hline Family & Botanical Name & Local Name & \begin{tabular}{|l|} 
Voucher \\
Number
\end{tabular} & $\begin{array}{l}\text { Plant } \\
\text { Part }\end{array}$ & Preparation & Therapeutic Uses \\
\hline Amaryllidaceae & Allium cepa Linn. & Alubosa & $\begin{array}{l}\text { LUH } \\
6942\end{array}$ & Bulb & $\begin{array}{l}\text { Cold } \\
\text { maceration }\end{array}$ & $\begin{array}{l}\text { Pelvic } \\
\text { inflammatory } \\
\text { disease }\end{array}$ \\
\hline Anarcadiaceae & $\begin{array}{l}\text { Spondias mombin } \\
\text { Linn. }\end{array}$ & Iyeye & $\begin{array}{l}\text { LUH } \\
1866\end{array}$ & $\begin{array}{l}\text { Leaf } \\
\text { and } \\
\text { fruit }\end{array}$ & $\begin{array}{l}\text { Decoction } \\
\text { and raw fruit. }\end{array}$ & $\begin{array}{l}\text { To ease labour and } \\
\text { childbirth }\end{array}$ \\
\hline Anarcadiaceae & $\begin{array}{l}\text { Mangifera indica } \\
\text { Linn. }\end{array}$ & Mangoro & $\begin{array}{l}\text { LUH } \\
1469 A\end{array}$ & $\begin{array}{l}\text { Leaf } \\
\text { and } \\
\text { bark }\end{array}$ & Decoction & Leucorrhea \\
\hline Annonaceae & $\begin{array}{l}\text { Uvaria chamae } \mathrm{P} . \\
\text { Beauv. }\end{array}$ & Eruju & $\begin{array}{l}\text { LUH } \\
3202\end{array}$ & Root & Decoction & $\begin{array}{l}\text { Amenorrhea, } \\
\text { Hyperprolactin- } \\
\text { emia }\end{array}$ \\
\hline Annonaceae & $\begin{array}{l}\text { Polyalthia } \\
\text { longifoila (Sonn.) } \\
\text { Thwaites }\end{array}$ & Ashoka & $\begin{array}{l}\text { LUH } \\
3562\end{array}$ & $\begin{array}{l}\text { Root } \\
\text { and } \\
\text { bark }\end{array}$ & Powder & $\begin{array}{l}\text { Menstrual } \\
\text { Disorders }\end{array}$ \\
\hline Annonaceae & $\begin{array}{l}\text { Xylopia } \\
\text { aethiopica } \\
\text { (Dunal) A. Rich. }\end{array}$ & Eeru & $\begin{array}{l}\text { LUH } \\
3863\end{array}$ & Fruit & Decoction & $\begin{array}{l}\text { To induce lactation } \\
\text { after child birth }\end{array}$ \\
\hline Apocynaceae & $\begin{array}{l}\text { Calotropis } \\
\text { procera Ait. }\end{array}$ & Bomubomu & $\begin{array}{l}\text { LUH } \\
3578\end{array}$ & $\begin{array}{l}\text { Leaf } \\
\text { and } \\
\text { stem }\end{array}$ & Decoction & $\begin{array}{l}\text { To increase milk } \\
\text { production after } \\
\text { childbirth }\end{array}$ \\
\hline Bignoniaceae & $\begin{array}{l}\text { Newbouldia } \\
\text { laevis Seem. }\end{array}$ & Akoko & $\begin{array}{l}\text { LUH } \\
3551\end{array}$ & Leaf & Decoction & $\begin{array}{l}\text { Menstrual } \\
\text { disorders, } \\
\text { infertility. }\end{array}$ \\
\hline Bignoniaceae & $\begin{array}{l}\text { Kigellia Africana } \\
\text { (Lam). Benth. }\end{array}$ & Pandoro & $\begin{array}{l}\text { LUH } \\
6097\end{array}$ & $\begin{array}{l}\text { Leaf } \\
\text { and } \\
\text { bark }\end{array}$ & Powder & $\begin{array}{l}\text { Breast pain, } \\
\text { Aphrodisiac }\end{array}$ \\
\hline Boraginaceae & $\begin{array}{l}\text { Heliotropium } \\
\text { indicum Linn. }\end{array}$ & $\begin{array}{l}\text { Atapari- } \\
\text { obuko }\end{array}$ & $\begin{array}{l}\text { LUH } \\
3006\end{array}$ & Root & Powder & Menorrhagia \\
\hline Cactaceae & $\begin{array}{l}\text { Opuntia dilenni } \\
\text { (Ker-Gawl.) Haw. }\end{array}$ & Oro agogo & $\begin{array}{l}\text { LUH } \\
7823\end{array}$ & Fruit & Decoction & Leucorrhea \\
\hline Curcubitaceae & $\begin{array}{l}\text { Colocynthis } \\
\text { citrullus (Linn) } \\
\text { Schrad. }\end{array}$ & Baara & $\begin{array}{l}\text { LUH } \\
6579\end{array}$ & Fruit & Tinctures & $\begin{array}{l}\text { Uterine fibroids, } \\
\text { Sexually } \\
\text { transmitted diseases }\end{array}$ \\
\hline Curcubitaceae & $\begin{array}{l}\text { Momordica } \\
\text { charantia Linn. }\end{array}$ & Ejirin-wewe & $\begin{array}{l}\text { LUH } \\
2736\end{array}$ & $\begin{array}{l}\text { Whole } \\
\text { plant }\end{array}$ & Decoction & $\begin{array}{l}\text { Infertility, } \\
\text { Dysmenorrhea }\end{array}$ \\
\hline Euphorbiaceae & $\begin{array}{l}\text { Euphorbia } \\
\text { laterifolia } \text { Schum } \\
\text { \&Thonn. }\end{array}$ & Enuopire & $\begin{array}{l}\text { LUH } \\
3288\end{array}$ & Bark & Powder & $\begin{array}{l}\text { Amenorrhea, } \\
\text { Veneral diseases }\end{array}$ \\
\hline Euphorbiaceae & $\begin{array}{l}\text { Jatropha curcas } \\
\text { Linn. }\end{array}$ & Botuje & $\begin{array}{l}\text { LUH } \\
3388\end{array}$ & Leaf & Leaf juice & Vaginal infections \\
\hline Euphorbiaceae & $\begin{array}{l}\text { Ricinus communis } \\
\text { Linn. }\end{array}$ & Ilara & $\begin{array}{l}\text { LUH } \\
4742\end{array}$ & $\begin{array}{l}\text { Leaf } \\
\text { and } \\
\text { seed }\end{array}$ & Tinctures & $\begin{array}{l}\text { To induce and ease } \\
\text { labour }\end{array}$ \\
\hline Euphorbiaceae & $\begin{array}{l}\text { Tetracarpidium } \\
\text { Conophorum } \\
\text { (Mull.Arg.) } \\
\text { Hutch.\& Dalziel }\end{array}$ & Awusa & $\begin{array}{l}\text { LUH } \\
5637\end{array}$ & Fruit & Cooked fruit & $\begin{array}{l}\text { Improves libido and } \\
\text { fertility. } \\
\text { It balances the } \\
\text { female hormones }\end{array}$ \\
\hline Fabaceae & $\begin{array}{l}\text { Acacia nilotica } \\
\text { Linn. }\end{array}$ & Booni & $\begin{array}{l}\text { LUH } \\
3146\end{array}$ & Bark & Decoction & $\begin{array}{l}\text { Aphrodisiac, } \\
\text { Gonorrhea, } \\
\text { Menstrual } \\
\text { problems. }\end{array}$ \\
\hline Fabaceae & $\begin{array}{l}\text { Baphia nitida } \\
\text { Lodd. }\end{array}$ & Iyerosun & $\begin{array}{l}\text { LUH } \\
3516\end{array}$ & Leaf & Infusion & $\begin{array}{l}\text { Amenorrhea } \\
\text { Dsymenorrhea }\end{array}$ \\
\hline Fabaceae & Delonix regia & Panseke & LUH & Leaf, & Decoction & Leucorrhea \\
\hline
\end{tabular}


Ethno-Gynaeacological Knowledge and Preliminary Phytochemical Screenings of Medicinal Plants used in Lagos State, Nigeria

\begin{tabular}{|c|c|c|c|c|c|c|}
\hline & (Hook.) Raf. & & 6524 & $\begin{array}{l}\text { bark } \\
\text { and } \\
\text { seed }\end{array}$ & & \\
\hline Fabaceae & $\begin{array}{l}\text { Pterocarpus osun } \\
\text { Craib. }\end{array}$ & Osun & \begin{tabular}{|l} 
LUH \\
3216
\end{tabular} & Bark & Powder & Amenorrhea \\
\hline Fabaceae & $\begin{array}{l}\text { Mucuna pruriens } \\
\text { Linn. }\end{array}$ & Werepe & $\begin{array}{l}\text { LUH } \\
4012\end{array}$ & Seed & Powder & $\begin{array}{l}\text { Aphrodisiac. } \\
\text { It enhances fertility. }\end{array}$ \\
\hline Hypoxidaceae & $\begin{array}{l}\text { Curculigo pilosa } \\
\text { (Schumach \& } \\
\text { Thonn.) Engl. }\end{array}$ & Epakun & $\begin{array}{l}\text { LUH } \\
4587\end{array}$ & Fruit & $\begin{array}{l}\text { Cold } \\
\text { maceration }\end{array}$ & $\begin{array}{l}\text { Uterine fibroids, } \\
\text { urinary tract } \\
\text { infections, } \\
\text { gonorrhea. }\end{array}$ \\
\hline Icacinaceae & $\begin{array}{l}\text { Rhaphiostylis } \\
\text { beninensis } \\
\text { (Hook.f.) Planch. }\end{array}$ & Itapara & $\begin{array}{l}\text { LUH } \\
5437\end{array}$ & Bark & Powder & Menorrhagia \\
\hline Iridaceae & $\begin{array}{l}\text { Gladiolus dalenii } \\
\text { Van. Geel. }\end{array}$ & Baka & $\begin{array}{l}\text { LUH } \\
6045\end{array}$ & Seed & Tinctures & Female sterility \\
\hline Lamiaceae & $\begin{array}{l}\text { Ocimum sanctum } \\
\text { Linn. }\end{array}$ & Efinrin-jije & $\begin{array}{l}\text { LUH } \\
5078\end{array}$ & Leaf & Leaf juice & $\begin{array}{l}\text { Constipation and } \\
\text { nausea. }\end{array}$ \\
\hline Liliaceae & $\begin{array}{l}\text { Aloe vera Linn } \\
\text { Burm. } f\end{array}$ & Ahon-Erin & $\begin{array}{l}\text { LUH } \\
3096\end{array}$ & Leaf & Leaf juice & $\begin{array}{l}\text { Aphrodisiac, } \\
\text { Amenorrhea, } \\
\text { Infections. }\end{array}$ \\
\hline Longaniaceae & $\begin{array}{l}\text { Anthocleista } \\
\text { djalolensis A. } \\
\text { Chev. }\end{array}$ & Sapo & $\begin{array}{l}\text { LUH } \\
3564\end{array}$ & Root & Powder & $\begin{array}{l}\text { Hormonal } \\
\text { Imbalance, } \\
\text { Annovulation. }\end{array}$ \\
\hline Loranthaceae & $\begin{array}{l}\text { Viscum album } \\
\text { Linn. }\end{array}$ & Afomo & $\begin{array}{l}\text { LUH } \\
2340\end{array}$ & $\begin{array}{l}\text { Whole } \\
\text { plant }\end{array}$ & $\begin{array}{l}\text { Infusion and } \\
\text { powder }\end{array}$ & $\begin{array}{l}\text { Uterine Fibroids, } \\
\text { Hormonal } \\
\text { imbalance, } \\
\text { Irregular } \\
\text { Menstruation }\end{array}$ \\
\hline Lythraceae & $\begin{array}{l}\text { Lawsonia inermis } \\
\text { Linn. }\end{array}$ & Laali & $\begin{array}{l}\text { LUH } \\
3837\end{array}$ & Root & Decoction & $\begin{array}{l}\text { Gonorrhoea, } \\
\text { leucorrhoea, } \\
\text { menorrhagia. }\end{array}$ \\
\hline Malvaceae & $\begin{array}{l}\text { Corchorus } \\
\text { olitorus Linn. }\end{array}$ & Ewedu & $\begin{array}{l}\text { LUH } \\
3767\end{array}$ & Leaf & Leaf juice & $\begin{array}{l}\text { To ease labour and } \\
\text { smooth delivery. }\end{array}$ \\
\hline Meliaceae & $\begin{array}{l}\text { Azadirachta } \\
\text { indica A. Juss. }\end{array}$ & Dongoyaro & $\begin{array}{l}\text { LUH } \\
5011\end{array}$ & $\begin{array}{l}\text { Leaf } \\
\text { and } \\
\text { bark }\end{array}$ & Decoction & Dysmenorrhea \\
\hline Moraceae & $\begin{array}{l}\text { Treculia africana } \\
\text { Decne. }\end{array}$ & Afon & $\begin{array}{l}\text { LUH } \\
4869\end{array}$ & $\begin{array}{l}\text { Whole } \\
\text { plant }\end{array}$ & Decoction & $\begin{array}{l}\text { Tubal blockage, } \\
\text { Miscarriage }\end{array}$ \\
\hline Moringaceae & $\begin{array}{l}\text { Moringa oleifera } \\
\text { Lam. }\end{array}$ & Ewe-igbale & $\begin{array}{l}\text { LUH } \\
4893\end{array}$ & Leaf & Infusion & $\begin{array}{l}\text { Hormonal } \\
\text { imbalance, } \\
\text { infertility }\end{array}$ \\
\hline Myristicaceae & $\begin{array}{l}\text { Staudtia stipitata } \\
\text { Warb. }\end{array}$ & Amuje & \begin{tabular}{|l|} 
LUH \\
6043
\end{tabular} & Bark & Decoction & $\begin{array}{l}\text { Amenorrhea } \\
\text { Dyspareunia }\end{array}$ \\
\hline Nymphaeaceae & $\begin{array}{l}\text { Nymphaea lotus } \\
\text { Linn. }\end{array}$ & Osibata & $\begin{array}{l}\text { LUH } \\
7236\end{array}$ & Leaves & Decoction & $\begin{array}{l}\text { Hyperprolactinem- } \\
\text { ia } \\
\text { Amenorrhea, } \\
\text { Annovulation }\end{array}$ \\
\hline Olacaceae & $\begin{array}{l}\text { Olax } \\
\text { subscorpiodiea } \\
\text { Oliv. }\end{array}$ & Ifon & $\begin{array}{l}\text { LUH } \\
3144\end{array}$ & Bark & Decoction & $\begin{array}{l}\text { Pelvic } \\
\text { inflammatory } \\
\text { disease }\end{array}$ \\
\hline Piperaceae & $\begin{array}{l}\text { Pepperomia } \\
\text { pellucida (Linn). } \\
\text { Kunth. }\end{array}$ & Rinrin & $\begin{array}{l}\text { LUH } \\
7283\end{array}$ & $\begin{array}{l}\text { Whole } \\
\text { plant }\end{array}$ & Infusion & $\begin{array}{l}\text { Irregular } \\
\text { Menstruation, } \\
\text { Dysmenorrhea }\end{array}$ \\
\hline Poaceae & Sorghum bicolor & Poporo & LUH & Whole & Decoction & Breast diseases, \\
\hline
\end{tabular}


Ethno-Gynaeacological Knowledge and Preliminary Phytochemical Screenings of Medicinal Plants used in Lagos State, Nigeria

\begin{tabular}{|c|c|c|c|c|c|c|}
\hline & Linn. & & 3068 & plant & & $\begin{array}{l}\text { Miscarriage, } \\
\text { Amenorrhea }\end{array}$ \\
\hline Poaceae & $\begin{array}{l}\text { Bambusa } \\
\text { vulgaris Linn. }\end{array}$ & Oparun & \begin{tabular}{|l} 
LUH \\
$1394 \mathrm{~A}$
\end{tabular} & $\begin{array}{l}\text { Leaves } \\
\text { and } \\
\text { young } \\
\text { shoots }\end{array}$ & Decoction & $\begin{array}{l}\text { Gonorrhea, } \\
\text { Aphrodisiac }\end{array}$ \\
\hline Poaceae & $\begin{array}{l}\text { Cynodon dactylon } \\
\text { Linn. }\end{array}$ & Kooko-igba & $\begin{array}{l}\text { LUH } \\
3045\end{array}$ & $\begin{array}{l}\text { Whole } \\
\text { plant }\end{array}$ & Decoction & $\begin{array}{l}\text { Amenorrhea, } \\
\text { Menopausal } \\
\text { symptoms, } \\
\text { Hot flashes }\end{array}$ \\
\hline Polygalaceae & $\begin{array}{l}\text { Securidaca } \\
\text { longipedunculata } \\
\text { Fresen }\end{array}$ & Ipeta & $\begin{array}{l}\text { LUH } \\
3150\end{array}$ & Root & Decoction & $\begin{array}{l}\text { Aphrodisiac, } \\
\text { Ovarian cyst }\end{array}$ \\
\hline Plumbaginaceae & $\begin{array}{l}\text { Plumbago } \\
\text { zeylanica Linn. }\end{array}$ & Inabiri & $\begin{array}{l}\text { LUH } \\
3203\end{array}$ & Bark & Powder & $\begin{array}{l}\text { Hormonal } \\
\text { imbalance, } \\
\text { Hyperpolactine-mia }\end{array}$ \\
\hline Rubiaceae & $\begin{array}{l}\text { Morinda lucida } \\
\text { Benth. }\end{array}$ & Oruwo & $\begin{array}{l}\text { LUH } \\
3819\end{array}$ & $\begin{array}{l}\text { Leaf } \\
\text { and } \\
\text { Bark }\end{array}$ & $\begin{array}{l}\text { Leaf juice and } \\
\text { bark } \\
\text { decoction }\end{array}$ & $\begin{array}{l}\text { Gonorrhea, } \\
\text { Fever during } \\
\text { childbirth }\end{array}$ \\
\hline Solanaceae & $\begin{array}{l}\text { Withania } \\
\text { somnifera Dunal. }\end{array}$ & Koroporo & $\begin{array}{l}\text { LUH } \\
7543\end{array}$ & Bark & Powder & $\begin{array}{l}\text { Amenorrhea, } \\
\text { Aphrodisiac }\end{array}$ \\
\hline Sterculiaceae & $\begin{array}{l}\text { Cola acuminata } \\
\text { Schott \& Engl. }\end{array}$ & Obi & $\begin{array}{l}\text { LUH } \\
6905\end{array}$ & Fruit & Decoction & $\begin{array}{l}\text { Endometriosis, } \\
\text { Amenorrhea }\end{array}$ \\
\hline Vitaceae & $\begin{array}{l}\text { Cissus populnea } \\
\text { Guill \& Perr }\end{array}$ & Ajara & $\begin{array}{l}\text { LUH } \\
6449\end{array}$ & $\begin{array}{l}\text { Leaf } \\
\text { and root }\end{array}$ & Decoction & $\begin{array}{l}\text { Sore } \\
\text { breast/Sexually } \\
\text { transmitted } \\
\text { infections } \\
\end{array}$ \\
\hline Vitaceae & $\begin{array}{l}\text { Cissus } \\
\text { quadrangularis } \\
\text { Linn. }\end{array}$ & Ogbakiiki & $\begin{array}{l}\text { LUH } \\
6458\end{array}$ & $\begin{array}{l}\text { Tuber } \\
\text { and } \\
\text { stem }\end{array}$ & Decoction & $\begin{array}{l}\text { Dysmenorrhea, } \\
\text { Urinary tract } \\
\text { infections. }\end{array}$ \\
\hline Zingberaceae & $\begin{array}{l}\text { Zingiber } \\
\text { officinale Roscoe. }\end{array}$ & Atale & $\begin{array}{l}\text { LUH } \\
4396\end{array}$ & Tuber & Decoction & $\begin{array}{l}\text { Morning sickness, } \\
\text { Nausea, } \\
\text { Pelvic } \\
\text { inflammatory } \\
\text { Disease }\end{array}$ \\
\hline Zygophyllaceae & Tribulus terrestris & Dagunro & $\begin{array}{l}\text { LUH } \\
4478\end{array}$ & $\begin{array}{l}\text { Whole } \\
\text { plant }\end{array}$ & Powder & $\begin{array}{l}\text { Aphrodisiac, } \\
\text { Hormonal } \\
\text { Imbalance }\end{array}$ \\
\hline
\end{tabular}

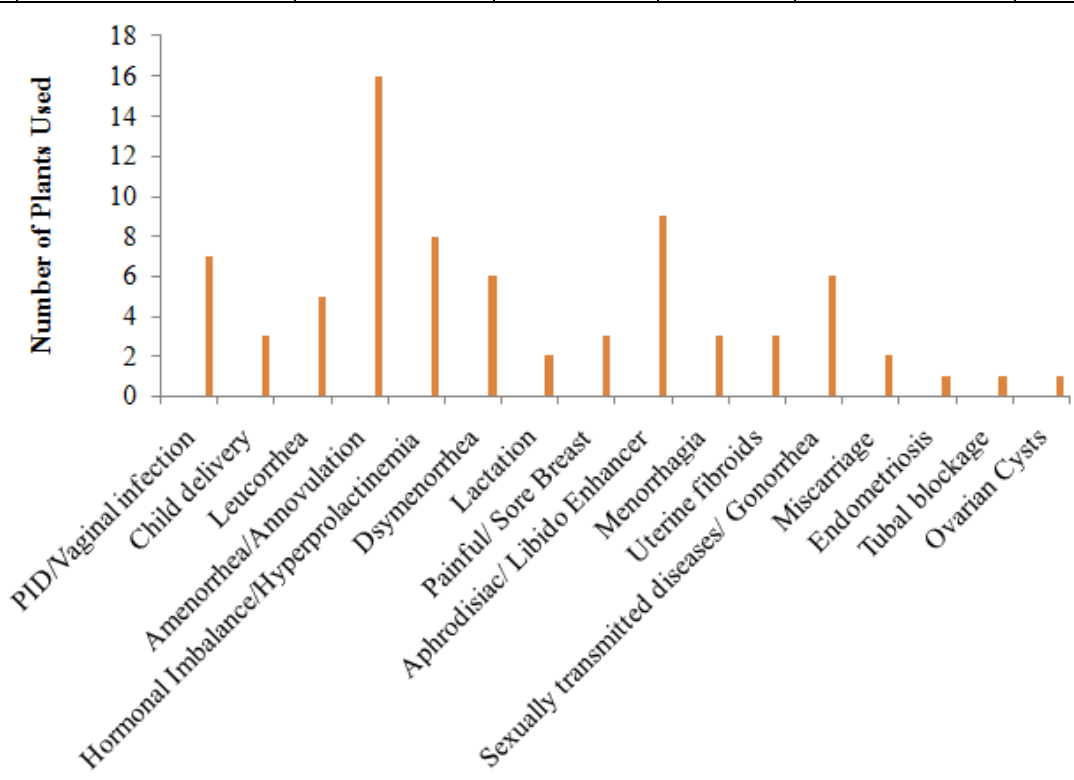

Gynaeacological Disorders

Figure2. Plants diversity used for a specific gynaeacological disorder in Lagos State, Nigeria 
Ethno-Gynaeacological Knowledge and Preliminary Phytochemical Screenings of Medicinal Plants used in Lagos State, Nigeria

Table3. Distribution within Families of Medicinal Plants Used For the Treatment of Different Gynaeacological Disorders in Lagos State, Nigeria

\begin{tabular}{|c|c|c|}
\hline $\mathbf{S} / \mathbf{N}$ & Family & Number of Species \\
\hline 1 & Amaryllidaceae & 1 \\
\hline 2 & Anarcadiaceae & 2 \\
\hline 3 & Annonaceae & 3 \\
\hline 4 & Apocynaceae & 1 \\
\hline 5 & Bignoniaceae & 2 \\
\hline 6 & Boraginaceae & 1 \\
\hline 7 & Cactaceae & 1 \\
\hline 8 & Curcubitaceae & 2 \\
\hline 9 & Euphorbiaceae & 4 \\
\hline 10 & Fabaceae & 5 \\
\hline 11 & Hypoxidaceae & 1 \\
\hline 12 & Icacinaceae & 1 \\
\hline 13 & Iridaceae & 1 \\
\hline 14 & Lamiaceae & 1 \\
\hline 15 & Liliaceae & 1 \\
\hline 16 & Longaniaceae & 1 \\
\hline 17 & Loranthaceae & 1 \\
\hline 18 & Lythraceae & 1 \\
\hline 19 & Malvaceae & 1 \\
\hline 20 & Meliaceae & 1 \\
\hline 21 & Moraceae & 1 \\
\hline 22 & Moringaceae & 1 \\
\hline 23 & Myristicaceae & 1 \\
\hline 24 & Nymphaeaceae & 1 \\
\hline 25 & Olacaceae & 1 \\
\hline 26 & Piperaceae & 1 \\
\hline 27 & Poaceae & 3 \\
\hline 28 & Polygalaceae & 1 \\
\hline 29 & Plumbaginaceae & 1 \\
\hline 30 & Rubiaceae & 1 \\
\hline 31 & Solanaceae & 1 \\
\hline 32 & Sterculiaceae & 1 \\
\hline 33 & Vitaceae & 2 \\
\hline 34 & Zingberaceae & 1 \\
\hline 35 & Zygophyllaceae & 1 \\
\hline
\end{tabular}

\subsection{Plant Habits, Parts used Modes of Preparation and Administration}

The percentage occurrence of the life forms (Plant habits) of the medicinal plants identified for the treatment of various gynaeacological disorders in Lagos State is as shown in Figure 3. The highest percentage of occurrence was observed in trees $(52 \%)$, followed by the herbs $(20 \%)$ while shrubs and creepers had $16 \%$ and $12 \%$ respectively.

The most commonly used plant parts were the leaves $(29.03 \%)$ and the bark $(22.58 \%)$ while the least commonly used plant part was the bulb (1.61\%) as shown in Figure 4.

Figure 5 is a representation of different methods of preparation of the medicinal plants used for the treatment of female gynaeacological disorders. Most of the medicinal plants identified were prepared by decoction $(48.08 \%)$ and powdered form $(23.08 \%)$ while the least method used was eating the plant raw $(1.29 \%)$. 
Ethno-Gynaeacological Knowledge and Preliminary Phytochemical Screenings of Medicinal Plants used in Lagos State, Nigeria

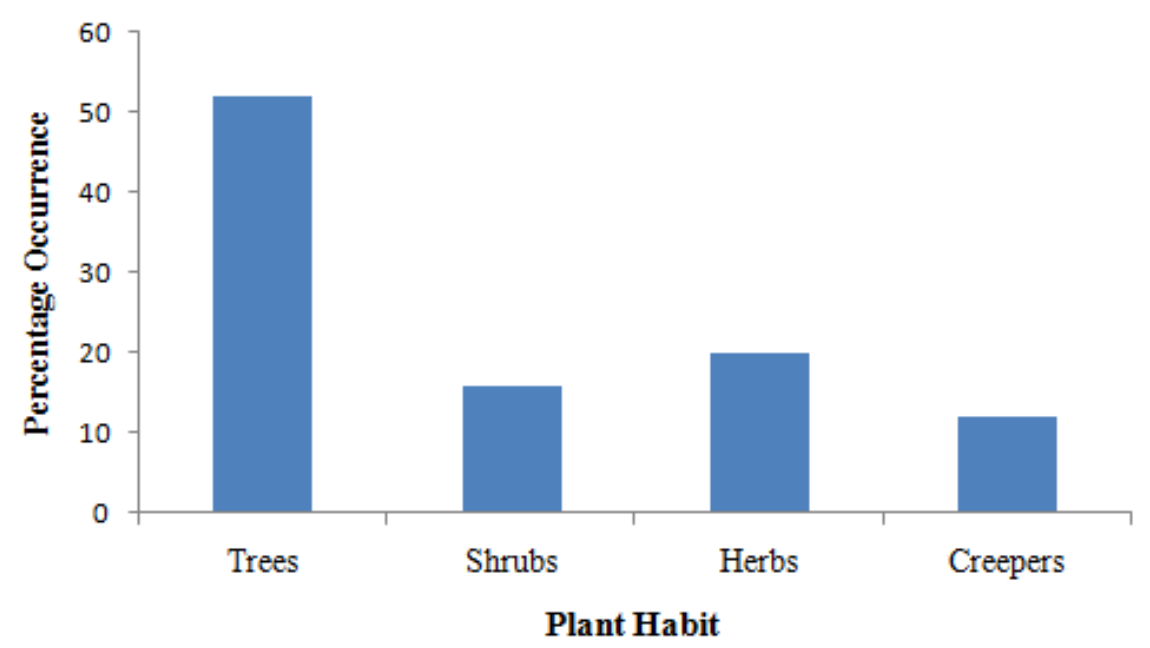

Figure3. Percentage occurrence of the plant life forms used for gynaeacological disorders in Lagos State, Nigeria

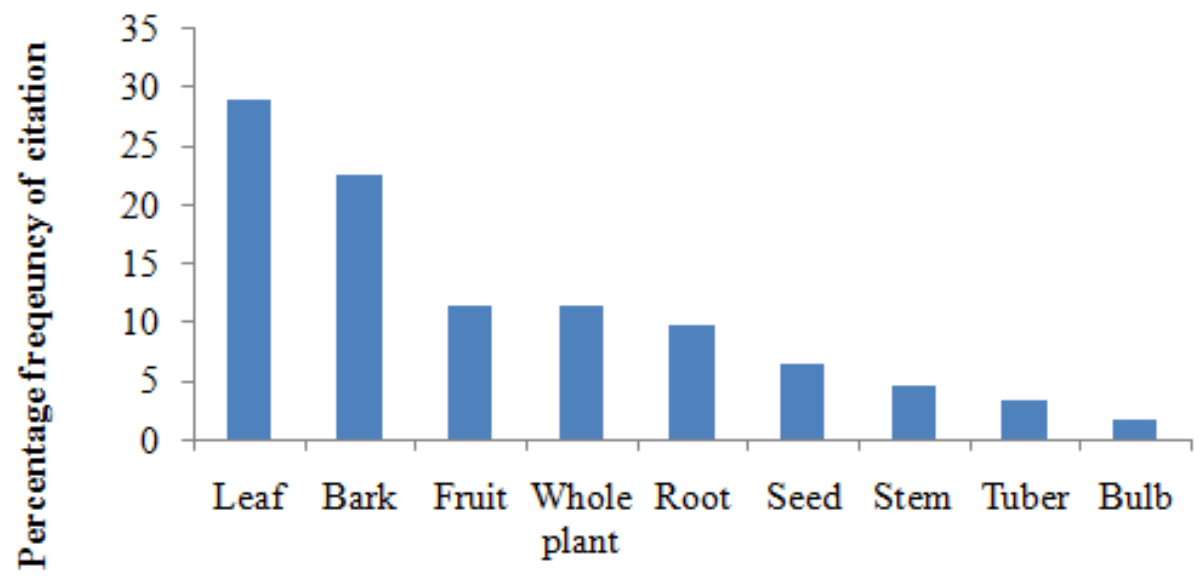

\section{Plant parts used}

Figure4. Percentage frequency of citation of plant parts used for gynaeacological disorders in Lagos State, Nigeria

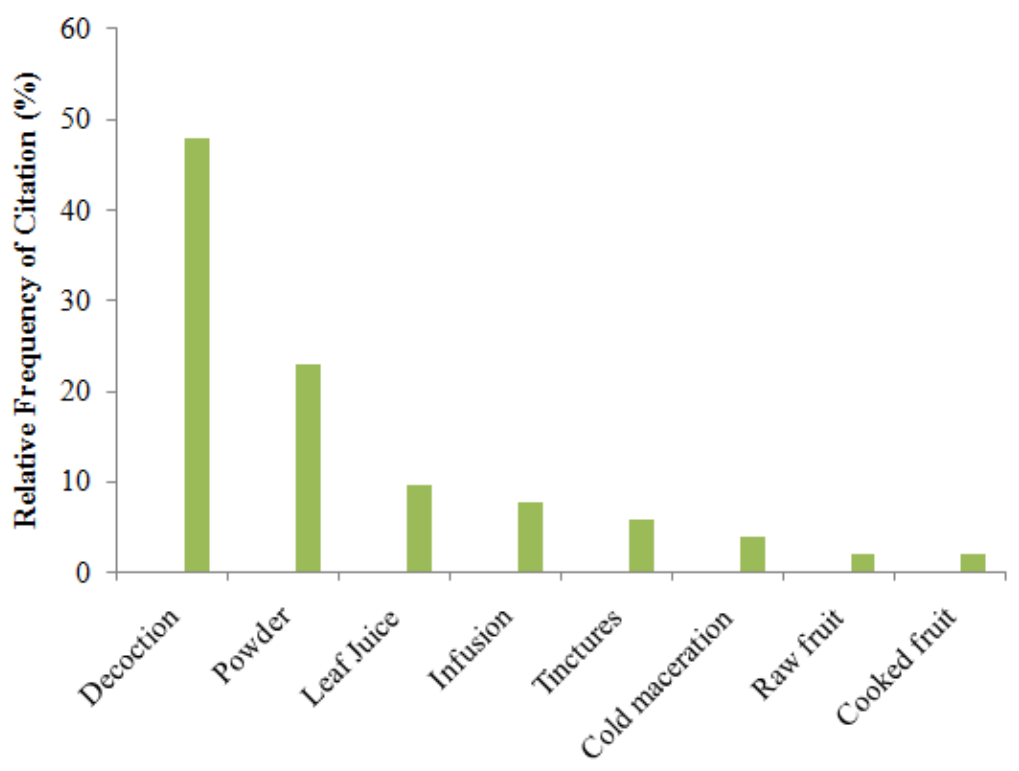

Modes of Preparation

Figure5. Percentage use of different modes of preparation of medicinal plants used for gynaeacological disorders in Lagos State 
Ethno-Gynaeacological Knowledge and Preliminary Phytochemical Screenings of Medicinal Plants used in Lagos State, Nigeria

\subsection{Qualitative Phytochemical Screenings}

The results of the preliminary phytochemical screenings of the aqueous extracts of all the medicinal plants used in folkloric medicine for the treatment of various gynaeacological disorders in Lagos State is shown in Table 4.The results of the preliminary phytochemical screenings showed that alkaloids were present in most of the plants except in Polyalthia longifolia, Acacia nilotica, Treculia africana, Bambusa vulgaris and Cynodon dactylon while tannins were absent only in Heliotropium indicum, Colocynthis citrullus, Euphorbia laterifolia, Jatropha curcas, Treculia africana and Sorghum bicolor. Phenols and flavonoids were present in all the plants while steroids were absent in Spondias mombin, Heliotropium indicum, Jatropha curcas, Baphia nitida, Azadiratcha indica and Bambusa vulgaris only. Phlobatannins were present only in ten (10) plant species; Xylopia aethiopica, Opuntia dilenni, Euphorbia laterifolia, Jatropha curcas, Staudtia stipitata, Nymphaea lotus, Sorghum bicolor, Plumbago zeylanica, Rhaphiostylis beninensis and Viscum album. Alkaloids have been widely reported to possess anti-inflammatory, analgesic and antimicrobial properties (Afolayan et al., 2013). The presence of the alkaloids in most of the medicinal plants identified may be responsible for the therapeutic actions against some of the gynaeacological disorders such as pelvic inflammatory disease, vaginal infections, urinary tract infections and painful or sore breasts. Phenols and flavonoids have been reported to have antioxidant, anticancer, anti-mutagenic, anti-inflammatory and antimicrobial properties. They easily donate electrons to free radicals thereby prevent the onset of degenerative diseases (Oki et al., 2002). Most diseases including some gynaeacological disorders are as a result of oxidative stress caused by the free radicals. Therefore, the presence of phenols and flavonoids in all the plants enumerated may suggest their ability to act as antioxidant against the free radicals caused by these disorders. Steroids have been reported to have aphrodisiac, antiviral and antibacterial properties. Oyedemi and Afolayan (2011) reported that steroids can improve sex hormones hence; they are useful in the treatment of sexual dysfunctions. Natural progesterone made from plant sterols called diosgenin has been reported to regulate hormonal imbalance and improves female hormones (Noguchi et al., 2006).

Table4. Qualitative Phytochemical Screenings of Medicinal Plants Used for the Treatment of Gynaeacological Disorders in Lagos State, Nigeria

\begin{tabular}{|c|c|c|c|c|c|c|c|c|c|c|}
\hline Plant & Alk & Tan & Phe & Sap & Ste. & Fla. & Proant & Antho & Gly & Phlo. \\
\hline Allium cepa Linn. & + & + & + & + & + & + & + & + & + & - \\
\hline Spondias mombin Linn. & + & + & + & + & - & + & - & - & + & - \\
\hline Mangifera indica Linn. & + & + & + & + & + & + & + & + & + & - \\
\hline Uvaria chamae P. Beauv. & + & + & + & + & + & + & + & - & + & - \\
\hline $\begin{array}{l}\text { Polyalthia longifoila } \\
\text { (Sonn.) Thwaites }\end{array}$ & - & + & + & + & + & + & - & + & - & - \\
\hline $\begin{array}{l}\text { Xylopia aethiopica (Dunal) } \\
\text { A. Rich. }\end{array}$ & + & + & + & - & + & + & + & + & - & + \\
\hline Calotropis procera Ait. & + & + & + & + & + & + & + & + & - & - \\
\hline Newbouldia laevis Seem. & + & + & + & + & + & + & + & - & - & - \\
\hline $\begin{array}{l}\text { Kigellia africana (Lam). } \\
\text { Benth. }\end{array}$ & + & + & + & + & + & + & - & - & + & - \\
\hline Heliotropium indicum Linn. & + & - & + & + & - & + & + & + & + & - \\
\hline $\begin{array}{l}\text { Opuntia dilenni (Ker-Gawl.) } \\
\text { Haw. }\end{array}$ & + & + & + & + & + & + & - & + & - & + \\
\hline $\begin{array}{l}\text { Colocynthis citrullus (Linn) } \\
\text { Schrad. }\end{array}$ & + & - & + & - & + & + & + & + & + & - \\
\hline Momordica charantia Linn. & + & + & + & + & + & + & - & - & + & - \\
\hline $\begin{array}{l}\text { Euphorbia laterifolia Schum } \\
\text { \&Thonn. }\end{array}$ & + & - & + & + & + & + & + & - & + & + \\
\hline Jatropha curcas Linn. & + & - & + & + & - & + & - & - & - & + \\
\hline Ricinus communis Linn. & + & + & + & + & + & + & - & - & + & - \\
\hline $\begin{array}{l}\text { Tetracarpidium } \\
\text { Conophorum (Mull.Arg.) } \\
\text { Hutch.\& Dalziel }\end{array}$ & + & + & + & + & + & + & + & - & + & - \\
\hline Acacia nilotica Linn. & - & + & + & + & + & + & + & - & - & - \\
\hline Baphia nitida Lodd. & + & + & + & + & - & + & + & + & - & - \\
\hline
\end{tabular}


Ethno-Gynaeacological Knowledge and Preliminary Phytochemical Screenings of Medicinal Plants used in Lagos State, Nigeria

\begin{tabular}{|c|c|c|c|c|c|c|c|c|c|c|}
\hline Delonix regia (Hook.) Raf. & + & + & + & - & + & + & - & - & + & - \\
\hline Pterocarpus osun Craib. & - & + & + & + & + & + & + & - & + & - \\
\hline Mucuna pruriens Linn. & + & + & + & - & + & + & + & + & - & - \\
\hline $\begin{array}{l}\text { Curculigo pilosa (Schumach } \\
\text { \& Thonn.) Engl. }\end{array}$ & + & + & + & + & + & + & + & + & + & - \\
\hline $\begin{array}{l}\text { Rhaphiostylis beninensis } \\
\text { (Hook.f.) Planch. }\end{array}$ & + & + & + & - & + & + & - & - & + & + \\
\hline Gladiolus dalenii Van. Geel. & + & + & + & - & + & + & - & + & - & - \\
\hline Ocimum sanctum Linn. & + & + & + & + & + & + & - & + & + & + \\
\hline Aloe vera (Linn) Burm.f & + & + & + & - & + & + & + & - & - & - \\
\hline $\begin{array}{l}\text { Anthocleista djalolensis A. } \\
\text { Chev. }\end{array}$ & + & + & + & - & + & + & + & - & - & - \\
\hline Viscum album Linn. & + & + & + & + & + & + & + & + & + & + \\
\hline Lawsonia inermis Linn. & + & + & + & + & + & + & + & - & + & - \\
\hline Corchorus olitorus Linn. & + & + & + & + & + & + & + & + & - & - \\
\hline Azadirachta indica A. Juss. & + & + & + & + & - & + & + & - & + & - \\
\hline Treculia africana Decne. & - & - & + & + & + & + & + & + & + & - \\
\hline Moringa oleifera Lam. & + & + & + & + & + & + & + & + & + & - \\
\hline Staudtia stipitata Warb. & + & + & + & + & + & + & - & + & + & + \\
\hline Nymphaea lotus Linn. & + & + & + & + & + & + & + & + & - & + \\
\hline Olax subscorpiodiea Oliv. & + & + & + & + & + & + & - & + & - & - \\
\hline $\begin{array}{l}\text { Pepperomia pellucida } \\
\text { (Linn). Kunth. }\end{array}$ & + & + & + & + & + & + & - & - & + & - \\
\hline Sorghum bicolor Linn. & + & - & + & + & + & + & + & - & + & + \\
\hline Bambusa vulgaris Linn. & - & + & + & + & - & + & + & - & - & - \\
\hline Cynodon dactylon Linn. & - & - & + & + & + & + & - & - & - & - \\
\hline $\begin{array}{l}\text { Securidaca } \\
\text { longipedunculata Fresen }\end{array}$ & + & + & + & - & + & + & + & - & + & - \\
\hline Plumbago zeylanica Linn. & + & + & + & + & + & + & - & - & + & + \\
\hline Morinda lucida Benth. & + & + & + & - & + & + & - & + & - & - \\
\hline Withania somnifera Dunal. & + & + & + & + & + & + & + & + & + & - \\
\hline $\begin{array}{l}\text { Cola acuminata Schott \& } \\
\text { Engl. }\end{array}$ & + & + & + & + & + & + & - & - & + & - \\
\hline $\begin{array}{l}\text { Cissus populnea Guill \& } \\
\text { Perr }\end{array}$ & + & + & + & + & + & + & - & + & + & - \\
\hline Cissus quadrangularis Linn. & + & + & + & + & + & + & + & - & - & - \\
\hline Zingiber officinale Roscoe. & + & + & + & + & + & + & + & - & + & - \\
\hline Tribulus terrestris Linn. & + & + & + & + & + & + & + & - & + & - \\
\hline
\end{tabular}

Character codes: Alk=Alkaloids, Tan=Tannins, Sap=Saponins, Phe=Phenols, Ste=Steroids, Fla=Flavonoids,

Proanto=Proanthocyanidins, Antho=Anthocyanidins, Gly= Cardiac Glycosides, Phlo=Phlobatannins, $+=$ Present, $-=$ Absent .

\section{CONCLUSION}

The rich plant diversity of the study area and the indigenous herbal knowledge of the respondents helped in the documentation of the medicinal plants used in the treatment of gynaeacological disorders in Lagos State. The type of phytochemicals present in the identified medicinal plants justified their usage in herbal medicine for the treatment of different gynaeacological disorders in the study area. This documentation would increase the indigenous knowledge of herbal medicine in Lagos State and Nigeria as a whole. It would also be helpful for the conservation of the identified medicinal plants. The identified medicinal plants could serve as precursors for the development of novel drugs for the treatment of various gynaeacological disorders.

\section{ACKNOWLEDGEMENT}

The authors acknowledge the Govan Mbeki Research Development Centre, University of Fort Hare for funding this study. 


\section{REFERENCES}

Afolayan, A.J., Sharaibi, O.J., Kazeem, M.I., 2013. Phytochemical analysis and in vitro antioxidant activity of Nymphaea lotus L. International Journal of Pharmacology 9: 297-304.

Akande, V.A., Hunt, L.P., Cahill, D.J., Caul, E.O., Ford, W.C., Jenkins, J.M., 2003. Tubal damage in infertile women: prediction using Chlamydia serology. Human Reproduction 18: 1841-1847.

Ali, T.S., Sami, N., Khujawa, A.K., 2007. Are unhygienic practices during menstrual, partum and postpartum periods risk factors for secondary infertility? Journal of Health Population and Nutrition 25: 189-194.

Al-Nahain, A., Jahan, R., Rahmatullah, M., 2014. Zingiber officinale: A potential plant against rheumatoid arthritis. Arthritis Volume 2014, Article ID 159089, 8 pages

Alzohairy, M.A., 2016. Therapeutic role of Azadirachta indica (Neem) and their active constituents in diseases prevention and treatment. Evidence-Based Complementary and Alternative Medicine. Volume 2016 (2016), Article ID 7382506, 11 pages.

Azima, K., Samina, J., 2002. Role of hyperprolactinemia in infertility. Pakistan Journal of Medical Research 41: 23-29.

Black, K.I., Fraser, I.S., 2012. The burden of health associated with benign gynecological disorders in lowresource settings. International Journal of Gynecology and Obstetrics 119: 72-75.

Colao, A., Di Sarno, A., Guerra, E., De Leo, M., Mentone, A., Lombardi, G., 2006. Drug insight: Cabergoline and bromocriptine in the treatment of hyperprolactinemia in men and women. Nature Reviews Endocrinology. 2:200-210

Dag, Z.O., Dilbaz, B., 2015. Impact of obesity on infertility in women. Journal of the Turkish-German Gynaeacological Association 16: 111-117.

Eggleston, K.N., Fuchs, V.R., 2012. The New Demographic Transition: Most Gains in Life Expectancy Now Realized Late in Life. Journal of Economic Perspectives 26: 137-156.

Freeman, M.E., Kanyicska, B., Lerant, A., Nagy, G., 2000. Prolactin: structure, function and regulation of secretion. Physiology Reviews 80 :1523-1631.

Fourman, L.T., Fazeli, P.K., 2015. Neuroendocrine causes of amenorrhea- An update. Journal of Clinical Endocrinology and Metabolism 100:812-824.

Harborne, J.B., 2005. Phytochemical Methods- A Guide to Modern Techniques of Plant Analysis, $3^{\text {rd }}$ ed.; Springer Pvt .Ltd, New Delhi, India. 302 Pp.

Hennessy, B.T., Coleman, R.L., Markman, M., 2009. Ovarian cancer. Lancet. 374:1371-1382

Ikechebelu, J.I., 2005. Prevalence of gynaeacological diseases in Nnewi, Nigeria. Nigerian Journal of Clinical Practice 8: 136-137.

Inyang-Etoh, P.C., Ogban, G.I., Inyang-Etoh, E.C., Useh, M.F., Etuk, S.J., 2009. Prevalence of Chlamydia trachomatis infection among Women attending infertility Clinics in Calabar, Nigeria. Nigerian Journal of Health and Biomedical Sciences 8: 1-2.

Jemal, A. Siegel, R., Xu, J., Ward, E., 2010. Cancer statistics. A Cancer Journal for Clinicians 60:277- 300

Kalaivani, K., 2009. Prevalence and consequences of anaemia in pregnancy. Indian Journal of Medical Research 130:627-633.

Llewellyn-Jones, D.L.,1998. Every Woman: A gynaeacological guide for life. Penguin Books Limited, New York, USA.

Mayo, J.L., 1998. Black cohosh and Chasteberry: Herbs valued by women for centuries. Clinical Nutrition Insights 6: 1-3.

Melmed, S., Casanueva, F.F., Hoffman, A.R., Kleinberg, D.L., Montori, V.M., Schlecte, J.A.,Wass, J.A.H., 2011. Diagnosis and Treatment of Hyperprolactinemia: An Endocrine Society Clinical Practice Guideline. The Journal of Clinical Endocrinology \& Metabolism, 96:2273-2288

Muthu,C., Ayyanar, M., Raja, N., Ignacimuthu, S., 2006. Medicinal plants used by traditional healers in Kancheepuram District of Tamil Nadu, India. Journal of Ethnobiology and Ethnomedicine 2:43

Nduche, M.U., Omosun, G., Okwulehie, I.C., 2015. Ethnobotanical Survey of Plants Used as Remedy for Fertility Conditions in Ebonyi State of Nigeria. Scholars Academic Journal of Biosciences 3:214-221.

Noguchi, E., Fujiwara, Y., Matsushita, S., Ikeda, T., Ono, M., Nohara, T., 2006. Metabolism of tomato steroidal glycosides in humans. Chemistry and Pharmacognosy Bulletin 54: 1312- 1314.

Odugbemi, T.O., Akinsulire, O.R., Aibinu, I.E., Fabeku, P.O., 2007. Medicinal plants useful for malaria therapy in Okeigbo, Ondo State, Southwest Nigeria. African Journal of Traditional, Complementary and Alternative Medicine 4: 191-198. 
Pandey S, Bhattacharya S, 2013. Impact of obesity on gynecology. Women health Review

Pattanayak, P., Behera, P., Das, D., Panda, S.K., 2010. Ocimum sanctum Linn. A reservoir plant for therapeutic applications: An overview. Pharmacognosy Review 47: 95-105.

Rustein, S.O., Shah, I.H., 2004. Infecundity, infertility and childlessness in Developing Countries. DHS Comparative Reports No 9. MEASURE DHS+ (Demographic and Health Surveys), US Agency for International Development (USAID), Maryland, USA and World Health Organization (WHO), Geneva, Switzerland.

Sadeghi, S., Mahmood, A., 2014. Ethno-gynecological knowledge of medicinal plants used by Baluch tribes, southeast of Baluchistan, Iran. Brazillian Journal of Pharmacognosy 24: 706-715.

Sadrefozalayi, S., Farokhi, F., 2014. Effect of the aqueous extract of Foeniculum vulgare (fennel) on the kidney in experimental PCOS female rats. Avicenna Journal of Phytomedicine 4: 114-224.

Schellenberg, R., 2001. Treatment for premenstrual syndrome with Vitex agnus-castus extract: prospective, randomised, placebo controlled study. British Medical Journal, 322:134-137.

Sharaibi, O.J., Ogundipe, O.T., Afolayan, A.J., Aworinde, D.O., 2014. Ethnobotanical survey and phytochemical analysis of medicinal plants used in the treatment of hyperprolactinemia in Lagos State, Nigeria. Journal of Medicinal Plants Research 8: 1284-1288.

Soladoye, M.O., Chukwuma, E.C., Sulaiman, O.M., Feyisola, R.T., 2014.Ethnobotanical survey of plants used in the traditional treatment of female infertility in Southwestern Nigeria. Ethnobotany Research and Applications 12: 81-90.

Sule, J.O., Erigbali, P., Eruom, L.,2008. Prevalence of Infertility in Women in a Southwestern Nigerian Community. African Journal of Biomedical Research 11: 225 - 227.

Tavasoli, F., Sharifian, J., Mazlom, R., 2002. Comparison of the effect of Mefenamic acid and Carum carvi on the severity of primary dysmenorrhea in Mashhad high- school students. Journal of Sabzevar University of Medical Sciences 8:4-9.

Titanji, V.P.K., Zofou, D., Ngemenya, M.N., 2008. The antimalarial potential of medicinal plants used for the treatment of malaria in Cameroonian folk medicine. African Journal of Traditional, Complementary and Alternative Medicine 5: 302-321.

Trease, G.E., Evans, W.C., 2002. Trease and Evans Pharmacognosy: A Physician's Guide To Herbal Medicine. 13th Edition. Bailliere, Tindall, London.

van Rijswijk, R.E., Vermoken, T.S., 2000. Drug therapy for gynaeacological cancer in older women. Drugs Aging 17: 31-32.

World Health Organization, 2002.General guidelines for methodologies on research and evaluation of traditional medicine. Switzerland.

Wuttke, W., Jarry, H., Christoffel, V., Spengler, B., Seidlova, W.D., 2003. Vitex agnus -castus: Pharmacology and indications. Phytomedicine 10:348-357.

Citation: O. Sharaibi, "Ethno-Gynaeacological Knowledge and Preliminary Phytochemical Screenings of Medicinal Plants used in Lagos State, Nigeria", International Journal of Medicinal Plants and Natural Products (IJMPNP), vol. 3, no. 2, pp. 6-18, 2017. http://dx.doi.org/10.20431/2454-7999.0302002

Copyright: (c) 2017 Authors. This is an open-access article distributed under the terms of the Creative Commons Attribution License, which permits unrestricted use, distribution, and reproduction in any medium, provided the original author and source are credited. 